\title{
Las incapacidades de aprendizaje organizacional y los estilos de aprendizaje en la industria papelera, cartonera y de celulosa de México
}

Organizational Learning Inabilities and Learning Styles in the Paper, Cardboard and Cellulose Industry in Mexico

As incapacidades de aprendizagem organizacional e os estilos de aprendizagem na indústria papeleira, cartonadora e de celulosa do México

\author{
José Gerardo Ignacio Gómez Romero* \\ Francisco Martín Villarreal Solís ${ }^{* \star}$ \\ María Deyanira Villarreal Solis ${ }^{\star \star \star}$
}

* Doctor en Administración de la Universidad Autónoma de Aguascalientes, México. Maestro en Administración de la Universidad Juárez del Estado de Durango. Contador Público de la Universidad Juárez del Estado de Durango. Profesor investigador en la Facultad de Economía, Contaduría y Administración de la Universidad Juárez del Estado de Durango (México). Correo electrónico: igomez@ujed

** Doctor en Administración de la Universidad Autónoma de San Luis Potosí, México, y Maestro en Contaduría Pública de la Universidad Autónoma de Nuevo León, México. Contador Público de la Universidad Juárez del Estado de Durango, México. Profesor investigador en la Facultad de Economía, Contaduría y Administración de la Universidad Juárez del Estado de Durango (México). Correo electrónico: fmvillasol@ujed.mx

*** Doctora en Administración de la Universidad Autónoma de San Luis Potosí, México, Maestra en Administración de la Universidad Juárez del Estado de Durango, México, y Licenciada en Administración de la Universidad Juárez del Estado de Durango, México. Profesora investigadora en la Facultad de Economía, Contaduría y Administración de la Universidad Juárez del Estado de Durango. Correo electrónico: devisol2000@yahoo.com.mx 
Fecha de recibido: 21 de agosto de 2014

Fecha de aprobado: 14 de enero de 2015

Doi: dx.doi.org/10.12804/rev.univ.empresa.29.2015.02

Para citar este artículo: Gómez Romero, J. G. I., Villarreal Solís, F. M., \& Villarreal Solís, M. D. (2015). Las incapacidades de aprendizaje organizacional y los estilos de aprendizaje en la industria papelera, cartonera y de celulosa de México. Universidad \& Empresa, 17(29), 35-62. Doi: dx.doi.org/10.12804/rev.univ.empresa.29.2015.02

\section{RESUMEN}

Esta investigación realiza un diagnostico en la industria de celulosa, cartón y papel en México concentrándose, en particular, en las incapacidades de aprendizaje organizacional y en los estilos de aprendizaje dominantes. Se indaga, además, la relación entre estas variables, de acuerdo con Yeung, Ulrich, Nason y Von Glinow (2000), y sus efectos en el aprendizaje organizacional. Se empleó un instrumento de medición basado en Yeung et al. (2000), adaptado por Gómez (2008). Las respuestas se analizaron con un modelo de regresión lineal que consideró las siete incapacidades de aprendizaje como variables predictoras de los cuatro estilos de aprendizaje. Se encontró evidencia estadística de una relación entre las incapacidades de aprendizaje y los estilos de aprendizaje organizacional en la industria estudiada.

Palabras clave: aprendizaje organizacional, industria papelera, cartonera y de celulosa de México, estilos de aprendizaje, incapacidades de aprendizaje.

\section{ABSTRACT}

This research consists of a diagnosis of the cellulose, cardboard and paper industry in Mexico and concentrates, in particular, on organizational learning inabilities and dominant styles of learning. The relationship between these variables, in accordance with Yeung, Ulrich, Nason and Von Glinow (2000), and their effects on organizational learning were studied. The measuring instrument used was the one based on Yeung et al. (2000), adapted by Gomez (2008). The replies were analyzed using a linear regression model that took into consideration the seven learning inabilities as predictive variables of the four styles of learning. Statistical evidence of a relationship between the learning inabilities and organizational learning styles in the industry studied was found.

Keywords: organizational learning, paper industry, cardboard and cellulose industry in Mexico, styles of learning, Learning inabilities.

\section{RESUMO}

Esta pesquisa realiza um diagnóstico na indústria de celulosa, cartão e papel no México se concentrando, em particular, nas incapacidades de aprendizagem organizacional e nos estilos de aprendizagem dominantes. Indaga-se, além disso, a relação entre estas variáveis, de acordo com Yeung, Ulrich, Nason y Von Glinow (2000), e seus efeitos na aprendizagem organizacional. Empregou-se um instrumento de medição baseado em Yeung et al. (2000), adaptado por Gómez (2008). As respostas se analisaram com um modelo de regressão lineal 
que considerou as sete incapacidades de aprendizagem como variáveis preditoras dos quatro estilos de aprendizagem. Encontrou-se evidência estatística de uma relação entre as incapacidades de aprendizagem e os estilos de aprendizagem organizacional na indústria estudada. Palavras-chave: aprendizagem organizacional, indústria papeleira, cartonadora e de celulosa do México, estilos de aprendizagem, incapacidades de aprendizagem.

\section{INTRODUCCIÓN}

El objetivo básico del presente estudio es indagar la posible relación entre las incapacidades de aprendizaje y los estilos de aprendizaje organizacional en la industria papelera, cartonera y celulosa de la República Mexicana. Se pretende también determinar qué incapacidad se presenta con más intensidad y, además, establecer cuál es el estilo de aprendizaje organizacional dominante en el sector industrial mencionado.

La Cámara del Papel (2013) de México, entre otras instituciones, ha destacado la importancia de este sector. Asevera que la producción mundial de esta industria ascendió a 400 millones de toneladas en 1999. Esto contra todos los pronósticos que apuntaban a una disminución del consumo de papel debido a la llegada y el auge de la era electrónica. De hecho, desde 1980 se observa un crecimiento constante en la tasa de producción en un promedio de 2,5\% anual. Además de sus ventajas económicas, el consumo de papel tiene el valor inherente a la función que juega en el registro y difusión de la información, de la historia y de la cultura.

Hoy en día la globalización, así como una creciente preocupación por la defensa y conservación del medio ambiente, han ejercido una presión importante sobre esta industria. Esto con el fin de tratar de garantizar la preservación de los recursos forestales utilizados en la fabricación del papel y sus derivados. Lo anterior ha conducido a los industriales a buscar alternativas de producción que no atenten contra el medio ambiente (Lenz, 1990).

Como consecuencia de lo anterior, en efecto, los industriales hacen un esfuerzo importante para convertir este tipo de empresas en organizaciones sustentables y amigables con el medio ambiente. En México, además de realizar adaptaciones técnicas e inversiones importantes dentro del proceso de producción, las compañías han cambiado durante los últimos ocho años su forma de producir, apoyándose en métodos que no contaminen y tratando de reciclar el papel que normalmente se desechaba. Gracias a esto el sector ha contribuido a 
desarrollar y a estructurar las actividades de recolección, superación y comercialización de los desperdicios que se aprovechan ahora como materia prima dentro de sus procesos de fabricación de papel (Redacción Teorema Ambiental, 2013).

\section{PLANTEAMIENTO DEL PROBLEMA}

El objetivo de la presente investigación se centra en analizar la relación entre las incapacidades de aprendizaje y los estilos de aprendizaje organizacional, así como en determinar cuál es la incapacidad y el estilo de aprendizaje dominante en plantas de fabricación de papel, cartón y celulosa de México.

La globalización es tal vez el fenómeno social, económico y político que más ha afectado en últimos tiempos la dinámica y la interacción entre las naciones y sus economías. Ello debido a que, además de cambiar la forma de convivencia y de intercomunicación de las personas en sociedad, este fenómeno modificó las maneras de hacer negocios y múltiples otras dimensiones vinculadas con la interacción entre los individuos, los grupos y las organizaciones (Gómez, 2008).

De acuerdo con Puerto (2010) efectivamente la economía global im- puesta por el fenómeno de la globalización presenta un horizonte de claroscuros para los individuos, las organizaciones, las instituciones, las empresas y las naciones. Estos deben aprender a manejarlo, a operar en él, si desean permanecer vigentes en sus ámbitos de competencia; de lo contrario, pueden caer en un vacío sin fondo en donde la expulsión de los mercados es el final no deseado.

Este paradigma abre un horizonte de nuevas condiciones que tienen que entender, equilibrar y compensar los individuos, las organizaciones y las empresas, a fin no verse rebasados por ellas. Para Gómez (2008), las empresas en la actualidad se tienen que enfrentar a la mezcla de todos estos factores. De hecho, por no saber hacerlo de manera adecuada, un número importante de compañías desaparece del mercado. Esto al no cumplir con los requerimientos que el entorno volátil y caprichoso les impone.

El tiempo en el que habitamos está marcado por la transición del paradigma de la sociedad industrial al de la sociedad del conocimiento, el cual está caracterizado, entre otros rasgos, por la globalización de las economías y de la política (Huntington, 2001). La sociedad del conocimiento, como es conocida esta nueva 
era, se caracteriza por la aparición continua de saberes nuevos y por el desarrollo permanente de las facultades intelectuales. Esto, como lo afirman Vázquez, Martínez y Monroy (2007), se ha concretado también en una acelerada caducidad de paradigmas y de las técnicas para observar la realidad.

Los cambios registrados según Toffler (1999) son acompañados de una alta competitividad, donde solo sobrevivirán las organizaciones que den respuesta a un entorno incierto, cambiante y volátil, es decir, aquellas que sean capaces de generar conocimiento (Yeung et al., 2000).

La generación de conocimiento es una característica que distingue a las organizaciones que aprenden, razón por la cual son llamadas 'inteligentes'. Según Choo (1999), el aprendizaje del ente social se produce cuando los miembros reaccionan a los cambios que se verifican en el medio ambiente, al detectar errores y corregirlos y, con ello, al modificar estrategias para sobrevivir.

Cuando las organizaciones no son capaces de reaccionar ante los cambios y las exigencias de un entorno volátil y cambiante, comprometen de algún modo su futuro. Esto se produce frecuentemente cuando las compañías no aprovechan el poten- cial que tienen, es decir, cuando no se benefician de su capacidad de aprendizaje. Esto sucede, entre otras razones, porque se enfrentan a las incapacidades o barreras de aprendizaje, las cuales les impiden aprovechar de mejor manera el potencial que poseen (Yeung et al. 2000).

\section{OBJETIVOS, PREGUNTAS DE INVESTIGACIÓN E HIPÓTESIS}

\subsection{Objetivos}

- Objetivo general: indagar la posible relación entre las incapacidades de aprendizaje y los estilos de aprendizaje organizacional en la industria papelera, cartonera y celulosa de la República Mexicana.

- Objetivos específicos:

- Determinar qué incapacidad se presenta con más intensidad en las empresas estudiadas.

- Establecer cuál es el estilo de aprendizaje organizacional dominante en el sector empresarial estudiado.

\subsection{Preguntas de investigación}

- ¿Existe una relación entre las incapacidades de aprendizaje y los estilos de aprendizaje organizacional en la la industria papelera, cartonera y celulosa de la República Mexicana? 
- En caso de existir, ¿qué incapacidades se presentan en mayor medida y más significativamente en los diferentes estilos de aprendizaje en la industria papelera, cartonera y de celulosa de la República Mexicana?

- ¿Qué estilo de aprendizaje organizacional es el dominante en el sector estudiado?

\subsection{Hipótesis}

Con base en lo indicado arriba se consideran las siguientes hipótesis:

- $\mathrm{H}_{1}$ : Existe una relación significativa entre las incapacidades de aprendizaje y los estilos de aprendizaje organizacional en la industria papelera, cartonera y celulosa de la República Mexicana.

- $\mathrm{H}_{2}$ : La incapacidad de aprendizaje que se presenta con mayor intensidad es la ceguera en las empresas estudiadas.

- $\mathrm{H}_{3}$ : El estilo de aprendizaje organizacional que se presenta con mayor intensidad en las empresas estudiadas es la mejora continua.

\section{JUSTIFICACIÓN}

La decisión de estudiar esta industria se tomó principalmente debido a la importancia creciente que ha demostrado tener este sector. En la actualidad, el mercado del sector papelero y sus productos derivados es de $\$ 11,5$ millones de dólares anuales, que equivale al $6,3 \% \mathrm{del}$ producto interno bruto (PIB) manufacturero y representan el 3,3\% del PIB industrial del país.

PriceWaterhouseCoopers (2011), en su reporte especializado anual, ha indicado que las empresas latinoamericanas experimentaron en 2010 el mayor crecimiento en las ventas en todo el mundo (del 28,8\%). Ello a causa del dominio de los productores de esta región en el mercado de la pulpa kraft blanqueada, una materia prima esencial para multitud de productos. Esta industria, a nivel global, generó en 2010 ventas por $\$ 339,1$ millardos de dólares, de acuerdo con la misma fuente.

Es necesario destacar que, en consonancia con las tendencias actuales de respeto al medio ambiente y responsabilidad social, la industria mexicana del papel y sus derivados ocupa el quinto lugar de los países recicladores de papel en el mundo. Esta reutiliza más de 4,7 millones de toneladas de papel en desuso. Ello representa el $87 \%$ de la materia prima fibrosa utilizada anualmente. Sin embargo, cabe destacar que mucha de esta fibra reciclable es importada de los Estados Unidos, dado que en nuestro país no es 
posible obtenerla por la falta de un manejo adecuado de los desechos.

En México la Cámara del Papel agrupa a la mayoría y a los más importantes productores nacionales de papel, cartón, corrugados y derivados, que producen el $98 \%$ de la producción nacional de esta industria. Son veintisiete empresas agremiadas en dicha institución y los datos registrados muestran que esta industria genera más de $64000 \mathrm{em}$ pleos directos y 235000 indirectos, a través de cincuenta y ocho plantas en veinte estados del país (Cámara del Papel, 2013). Según esta institución, el 90\% de las empresas asociadas cuentan con sistemas de tratamiento de aguas residuales. Ello les permite reutilizar el agua dentro de sus procesos y tratarla de manera controlada, antes de su destino final. La industria está implementando además sistemas de autogeneración de energía eléctrica. De hecho, actualmente se lleva un avance de más del $20 \%$ en el sector, lo que se traduce en ahorros sustantivos, pero, sobre todo, en garantizar la menor contaminación posible.

La industria del papel es hoy una de las mayores 'industrias de captación', en emisiones de $\mathrm{CO}_{2}$ equivalente, dada la naturaleza de sus actividades, la reforestación, el uso y el reciclaje(Aldrich, Llauro, Puig, Mut- je, \& Pelach, 2013). Por todo esto, el papel que se comercializa en México es en realidad un producto sustentable, dado que se controla el origen de las fibras, el consumo de energía, agua y otros recursos.

Redacción Teorema Ambiental (2013) sostiene que la industria ha invertido más de $\$ 6000$ millones de dólares para la modernización de su planta productiva y para el desarrollo de nuevos usos sustentables. Más de dos tercios del consumo del papel en México es generado por la industria nacional, satisfaciendo así las necesidades crecientes de la población. Además, se hacen esfuerzos por promover la cultura de la separación de residuos sólidos, los cuales son reutilizables para el papel, pero también para muchas otras cadenas productivas.

La industria fabricante de celulosa, papel y cartón es creadora importante de empleos directos e indirectos. Los estados en donde se encuentran sus plantas productivas son Baja California, Chihuahua, Coahuila,Hidalgo, Durango, Guanajuato, Jalisco, Michoacán, Morelos, Nuevo León, Puebla, Oaxaca, Querétaro, San Luis Potosí, Sonora, estado de México, Tlaxcala, Veracruz y el Distrito Federal.

En cuanto al posicionamiento internacional, de acuerdo con De la 
Madrid (2011), dos empresas mexicanas se encuentran entre las cien mayores productoras de papel del mundo, con ventas combinadas de casi \$3000 millones de dólares. El $38 \%$ del total de ventas en el planeta se genera en América del Norte, el $34 \%$, en Europa y el $18 \%$, en Asia. Latinoamérica contribuía en 2008 con el $4 \%$ del total de las ventas a nivel mundial. El 6\% restante se originó en África y Oceanía.

\section{REVISIÓN DE LITERATURA}

De unos años a la fecha sociólogos, economistas, administradores yotros profesionales hablan de la Sociedad del Conocimiento, para referirse, entre otras cuestiones, a los grandes cambios que se han dado en los albores del siglo XxI. Esta sociedad se distingue por la aparición continua de nuevos saberes, por la emergencia de formas nuevas de ver y de concebir las cosas, acompañado de una aceleración inusitada en el proceso de cambio de los paradigmas que imperaban con anterioridad. Huntington (2001) indica que los tiempos que vivimos están marcados justamente por la transición del paradigma de la sociedad industrial al de la sociedad del conocimiento, el cual está enmarcado, entre otros fenómenos, por la globalización de las economías y de la política.
La globalización como proceso económico, social y cultural es una herencia fundamental del siglo xx. Este hace que todas las empresas, de algún modo, estén inmersas en un entorno cada vez más incierto y volátil (Bravo, 2012). De hecho, se espera que su mayor esplendor tenga lugar en el tercer milenio. La transformación de la cual somos testigos trae como consecuencia, entre otras cosas, una alta competitividad en donde solo sobreviven las organizaciones que sepan dar respuesta a un entorno incierto, cambiante y volátil, aquellas que, de acuerdo con Yeung et al. (2000), sean capaces de generar conocimiento.

De todo esto se puede inferir que el aprendizaje se ha convertido en la actualidad en una clave esencial para que las organizaciones sobrevivan y se conviertan en instituciones que aprenden. Aunque, como afirman Yeung et al. (2000) y Hernández y Martí (2006), esto no es una panacea para enfrentar el conjunto de debilidades de la organización, sí permite que se proporcione una mejor respuesta a su entorno.

El uso del conocimiento como la nueva fuente de producción de riqueza está cambiando por completo la estructura de la sociedad y, por consiguiente, de las propias organizaciones. Valdés (2002) afirma que 
antes el saber se aplicaba al ser, pero que ahora, en la nueva era, se aplica al hacer. Se piensa por lo general que aquellas instituciones que no sean capaces de generarlo están condenadas a desaparecer. La creación de conocimiento es una característica que distingue a las organizaciones que aprenden, por lo que son llamadas discentes o inteligentes.

El conocimiento en la actualidad se destaca como un factor clave para obtener competitividad en las organizaciones. Probst, Rauby Romhardt (2001) aconsejan que estas se deban de orientar a la generación de nuevas habilidades, productos, ideas y procesos más eficaces. También aceptan que el desarrollo del conocimiento es un pilar que complementa la adquisición del propio saber.

En este punto, con base en lo indicado, es posible concluir que la generación de conocimiento organizacional es la capacidad de la compañía para producir nuevos saberes a partir de sus empleados, diseminarlos entre todo su personal y materializarlos en nuevos productos, servicios y sistemas o en el perfeccionamiento de los ya existentes.

De acuerdo con Valdés (2002), uno de los aprendizajes más importantes de la última década consiste en que cuando un paradigma nuevo se esta- blece, el éxito pasado se convierte en el peor enemigo. Esto dado que los cambios externos son más rápidos que la capacidad de las organizaciones para entenderlos y adoptarlos en el contexto de una sociedad globalizada (conocida también como la sociedad de la posmodernidad). Choo (1999) y Beazley, Boenisch y Harden (2003), por su parte, coinciden en afirmar que el aprendizaje del ente social se produce cuando los empleados de la organización reaccionan ante los cambios que se verifican en el medio ambiente. Ello al detectar errores y corregirlos, así como al modificar estrategias, supuestos o normas. Lo anterior es vital para la productividad, la innovación y el desempeño de las empresas y de sus empleados.

En la actualidad, las organizaciones se ven enfrentadas a aprender a capitalizar su experiencia para poder sobrevivir. Estas requieren identificar que el único capaz de crear conocimiento es el ser humano, quien, al insertarse en las empresas, hace que estas difundan conocimiento organizacional, necesario en la actualidad para subsistir. Necesitan poder vislumbrar que dicho aprendizaje, además de garantizarles su permanencia en los mercados, debe impactar en su crecimiento como institución (Carrión, 2007). 
Las organizaciones aprenden a través de dos fuentes básicas:

1. La experiencia directa, en donde se aprende como resultado de los propios actos y reflexiones.

2. La experiencia de terceros, que se basa en lo que se aprende de otros, como asesores, proveedores o competidores, además de incluir el aprendizaje por observación.

Se aprende de manera directa cuando el ambiente en donde se desenvuelve la empresa cambia de repente y se compite mediante la innovación; por el contrario, cuando el ambiente es estable y se rivaliza con costos y bienes de servicio, se aprende principalmente a través de la experiencia de terceros.

De acuerdo con Yeung et al. (2000), las personas y las organizaciones tienen, en lo fundamental, cuatro estilos para aprender. Estos son:

1. Experimentación: En donde se aprende probando ideas nuevas y adoptando una posición receptiva del entorno.

2. Adquisición de competencias: Consiste en que las personas y los equipos adquieran competencias nuevas. Se centra en la experiencia de terceros.

3. Marcas de referencia (benchmarking): Se aprende averiguando cómo funcionan otros y a partir de ahí se trata de adaptar estos conocimientos.

4. Mejoras continuas: En donde se aprende mejorando constantemente lo que ya se hace.

Dichos estilos solo representan tipos ideales de aprendizaje pues, así como las personas jamás se ciñen a un solo tipo de temperamento cuando se desenvuelven en su vida cotidiana, a pesar de que tengan un temperamento dominante, las empresas y los individuos rara vez aprenden exclusivamente siguiendo un único estilo. Yeung et al. (2000) señalan también que las organizaciones no deben limitarse a solo uno de estos, sino, al contrario, de ser posible, deben tratar de utilizarlos todos en alguna etapa de la vida.

Etkin (2007) y Gómez (2008) coinciden en afirmar que las organizaciones aprenden cuando las ideas y los conocimientos que generan sus empleados son compartidos más allá de los límites del espacio físico de la organización. También es necesario entender que el aprendizaje pasa de algún modo por muchos 'pequeños fracasos', algunos de los cuales pueden llevar a la compañía a la muerte. Esto a menos que esos fracasos sean 'inteligentes', es decir, que permitan aprender de ellos y fortalezcan la empresa para 
enfrentar la realidad de otra manera en un futuro.

Nonaka y Takeuchi (2000) afirman que la creación de conocimiento en la organización es la capacidad de esta para generar nuevos saberes, diseminarlos entre los miembros de la empresa y materializarlos en productos, servicios y sistemas. Estos autores coinciden con Senge (1993) y Choo (1999) al afirmar que para construir una organización que aprende los ejecutivos deben:

1. Adoptar un pensamiento sistémico.

2. Fomentar la maestría personal de sus propias vidas.

3. Sacar a flote y desafiar los modelos mentales prevalecientes.

4. Crear una visión compartida.

5. Facilitar el aprendizaje de equipo.

Una organización que aprende es un lugar donde las personas descubren constantemente cómo crear su realidad y cómo pueden cambiarla. Lo que aprende una compañía inicia con lo que aprenden las personas, pero no se limita a esto. Las organizaciones inteligentes están en una posición ventajosa para sobrevivir y triunfar, en particular si se enfrentan a un entorno cambiante e incierto. Pueden cumplir de mejor manera con su misión y alcanzar su visión en comparación con las empresas a las que no les interesa generar conocimiento (Gómez, 2008).

Es posible definir la capacidad de aprendizaje de las organizaciones como el potencial dinámico de creación, asimilación, difusión y utilización del conocimiento por medio de numerosos flujos del mismo, que hacen posible la formación y evolución de las memorias organizacionales de saber que capacitan a las organizaciones y sus agentes para actuar intencionalmente en entornos cambiantes (Prieto, 2004).

Las organizaciones, en general, producen conocimiento en mayor o menor medida, sean conscientes de esto o no y estén preparadas para ello o no. Esto tiene como consecuencia que, al enfrentarse a un entorno volátil, complejo y competitivo como el actual, muchas de estas fracasen, pues en la presente era del conocimiento, como sugieren Yeung et al. (2000), solo sobreviven las organizaciones que estén preparadas, aquellas que, entre otras competencias, sean capaces de generar conocimiento organizacional y aplicarlo.

Yeung et al. (2000) argumentan que no es casualidad que la mayoría de organizaciones aprendan mal. Estos coinciden con Argyris (1993) al considerar que la incipiente formación administrativa de los gerentes 
y propietarios de los negocios, acarrea necesariamente deficiencias en la generación del conocimiento organizacional (CO). A estas deficiencias se las reconoce como 'barreras o incapacidades de aprendizaje'. Estas incapacidades impiden que el aprendizaje sea efectivo. Son como una enfermedad silenciosa y, aunque no provocan aparentemente ningún síntoma, evitan que algunos procesos se realicen de manera asertiva. Una vez han invadido la organización, terminan por hacerla inoperante, por hacer que sea incapaz de reaccionar ante los cambios del entorno. Estas también frenan la capacidad de rehacerse a través de adecuados procesos de realimentación y de aprender de los errores que cometen, como consecuencia, no consiguen dar una respuesta adecuada a las demandas de los consumidores.

El tema de las barreras o incapacidades de aprendizaje se presenta, por lo general, como algo secundario y, en consecuencia, es un asunto que casi no es tratado; sin embargo, la importancia de estudiarlas radica en que si no se identifican a tiempo, si no se superan, las organizaciones ciertamente podrían continuar generando de algún modo aprendizaje organizacional, pero este será deficiente y estará plagado de problemas y errores, desde su elaboración hasta su generalización. El concepto de barreras o incapacidades de aprendizaje se refiere entonces a las condiciones que dificultan u obstaculizan el aprendizaje en el ámbito de las organizaciones y de los negocios y que impiden que, independientemente del estilo de aprendizaje presente en la organización, a esta le sea difícil o imposible llegar a generar conocimiento.

A partir de la revisión de la literatura realizada se encontró que, en general, los autores que trabajan el tema de estas barreras, coinciden en los efectos que ellas provocan, aunque algunos las desglosan más que otros. A continuación se hace un recorrido sintético por algunos de los principales autores que han tratado este tema y al final se presentan las incapacidades de aprendizaje que distinguen Yeung et al. (2000). En estas, en lo fundamental, se ha basado el presente estudio.

Se encuentra, en primer lugar, que Senge (1993) ha planteado siete barreras. Estas son:

1. Yo soy mi puesto: Cuando las personas en una organización solo se preocupan por las tareas propias de su puesto y no sienten ninguna responsabilidad por el efecto de su actividad en otras partes del negocio. Esto produce una falta de visión sistémica que 
impide los intercambios entre las personas y, con ello, se dificulta el aprendizaje organizacional.

2. El enemigo externo: Es la práctica permanente de culpar de los errores a los demás, así como a factores externos aparentemente extraordinarios y no reconocer nunca los propios errores. Esto, por supuesto, imposibilita su corrección.

3. La ilusión de hacerse cargo: Se traduce en acciones reactivas, que son impensadas, buscando solucionar problemas. Estas concluyen en soluciones fallidas que terminan bloqueando el aprendizaje.

4. La fijación en los hechos: Prestar atención solo a lo resolver lo inmediato, sin ir más allá de los problemas visibles e ignorando aquellos procesos soterrados y a largo plazo. Esta miopía condena a la organización a la reactividad, imposibilitando la adopción de comportamientos proactivos y de soluciones creativas a los problemas.

5. La parábola de la rana hervida: Es la incapacidad de ver los cambios lentos y graduales, siendo solamente sensibles a los cambios rápidos y bruscos. El resultado es la insensibilidad a las alternaciones sutiles.

6. La ilusión de que se aprende con la experiencia: Múltiples organi- zaciones asumen que se aprende mejor de la experiencia. Esto sin tener en cuenta que, en ocasiones, no es posible experimentar directamente las consecuencias de determinados actos o decisiones importantes, pues algunos de estos tienen consecuencias a muy largo plazo y, en no pocos casos, estas incluso son imposibles de predecir en su totalidad.

7. El mito del equipo administrativo: Parte del pensamiento erróneo de que el equipo directivo está formado por personas capacitadas para resolver todos los problemas. Esto puede obstaculizar claramente el aprendizaje, en particular, en aquellos casos en los que el equipo directivo no tiene la capacidad para resolverlos.

Levitt y March (1988), por su parte, identifican dos barreras que dificultan el aprendizaje, a saber:

1. El aprendizaje supersticioso: Parte del principio de que determinadas rutinas o formas de hacer conducen al logro de resultados exitosos, sin tener ninguna prueba de que en realidad esto sea así. Esta creencia impide la revisión de lo realizado.

2. Las trampas de la competencia: La habilidad que se adquiere al desarrollar una actividad de manera frecuente conduce a pensar a 
quien la realiza que es un experto. Esta actitud dificulta el aprendizaje, pues las personas se muestran reacias a abandonar rutinas que han logrado dominar. Ello por el miedo del retorno a cero, de tener que volver a comenzar, haciendo de este modo difícil que se aprendan cosas nuevas.

Geranmayeh (1992) también distingue dos incapacidades a las que identifica de la siguiente manera:

1. El mito de la infalibilidad: Tiene como fundamento la idea de que los directivos jamás cometen errores. De hecho, como se observa, esta se asemeja al mito del equipo administrativo planteado por Senge (1993).

2. Matar al mensajero: Se refiere al pensamiento de que es usual que se castigue al portador de una mala noticia. Esto inhibe que se pueda hablar de los errores y fracasos en la organización, lo que bloquea el aprendizaje a partir de las fallas en las que se ha incurrido.

Por su lado, Argyris (1993) identifica y describe cuatro barreras. Estas son:

1. La incompetencia competente: Se refiere a las acciones que buscan solucionar algo, pero tienen un efecto contraproducente.
Esto, en particular, por el hecho de apegarse para su solución a marcos prestablecidos.

2. Las rutinas defensivas: Se fundan en la idea de que "aquí no pasa nada y todo está bien", en el hecho de no reconocer los errores y sus consecuencias. Cuando esto se presenta los individuos son incapaces de aprender, pues no se admiten los errores para poder corregirlos y reorientar las acciones.

3. El autoengaño: Se traduce en una ceguera que impide ver lo que está mal, llegando el actor a engañarse incluso a sí mismo y a culpar a otras personas de los errores cometidos.

4. El malestar organizativo: Es una consecuencia del autoengaño. Se da al no aceptar las incongruencias y los errores, lo que termina por crear un clima de malestar generalizado. El personal no se siente bien pero, al mismo tiempo, es incapaz de cambiar, pues no se dan las condiciones necesarias para ello. Esto termina con la generación de críticas, la magnificación de lo negativo y la minimización de lo positivo.

Otros investigadores, entre los que se encuentran, Probst y Büchel (1995), identifican también algunas incapacidades de aprendizaje. Estos dos autores, en particular, coinciden con 
el planteamiento realizado por Argyris (1993), pero agregan a las planteadas por estos dos más, a saber:

1. Privilegios y tabúes: Generalmente en todas las empresas existen algunas personas que poseen ciertos privilegios, quienes bloquean el aprendizaje al resistirse a renunciar a los mismos cuando se presentan cambios en la organización. Esto termina cuestionando todo cambio que llegue a tratar de romper su estatus personal y sus condiciones actuales.

2. Patología de la información: Se presenta cuando no se saben manejar adecuadamente los datos con los que se cuenta para tomar decisiones de manera apropiada.

Lounamaa y March (1987) plantea de manera genérica "el dilema del aprendizaje", que consiste en pensar que la resolución de corto de problemas resolverá el rumbo a largo plazo, dejando el futuro al azar por la resolución de la inmediatez.

Por otro lado, Watkins \& Marsick (1993) también han contribuido a esta temática. Ello a través de la identificación, en particular, de tres barreras:

1. La incapacidad aprendida: Resulta de la pasividad y la desmo- tivación de los individuos ante las oportunidades de aprendizaje. Esto como producto de que la gente no es tomada en cuenta en la toma de decisiones y al hecho de no querer asumir responsabilidades.

2. La visión de túnel: Es la incapacidad de descubrirse desde un punto de vista sistémico y de actuar en consecuencia. Las personas son conscientes de su propia perspectiva, pero no entienden que hay competencia a su alrededor y que es necesario actuar en consecuencia. Esto si se quiere que el negocio sobreviva.

3. El aprendizaje truncado: Ocurre en aquellas organizaciones que no han asumido realmente la necesidad de aprender y realizan operaciones de "maquillaje" orientadas a incorporar pequeños cambios, pero sin llegar a implicarse en procesos de cuestionamiento profundo.

Por último, se destaca la propuesta que hacen Yeung et al. (2000). Estos autores plantean siete incapacidades. Las cuatro primeras impiden la generación del conocimiento y las tres restantes se oponen a su generalización:

1. Ceguera: Impide evaluar correctamente las amenazas y oportunidades del entorno, dejando de lado un sinfín de oportunidades. 
2. Candidez: Se presenta cuando hay deficiencias en el análisis de las situaciones y la generación de soluciones pues, ante situaciones complejas, se aplican las reglas "de cajón", sin que se realice en realidad un análisis cuidadoso. Se opta entonces por soluciones fáciles ante problemas difíciles.

3. Homogeneidad: Solo se toma en cuenta el punto de vista del dueño o gerente, quien es "omnisciente y experto en todo". Se olvida que, cuantas más interpretaciones se encuentren en una organización, mayores podrán ser las oportunidades de aprendizaje. Esto, en particular, cuanto el ambiente es más complejo.

4. Acoplamiento estrecho: La inflexibilidad y la no adaptabilidad es la tónica, pues se deriva de la realización de análisis de la realidad ingenuos e incompletos.

5. Parálisis: Impide tomar medidas o implantar procedimientos nuevos, no porque no se sepa en un determinado momento cómo realizarlos, sino porque se emplea demasiado tiempo en el análisis de las cosas.

6. Aprendizaje de supersticiones: Plantea la incapacidad para interpretar correctamente el significado de la experiencia, además de tratar de culpar a terceras personas de lo malo que ocurre en el negocio, difundiendo ideas equivocadas acerca de la situación.
7. Difusión deficiente: Se presenta cuando las personas aprenden cosas nuevas, pero no las difunden a otras y, como consecuencia, no se da el aprendizaje colectivo.

\section{METODOLOGÍA}

Este estudio es correlacional, ya que busca establecer la relación entre variables. De acuerdo con lo planteado por Hernández, Fernández y Baptista (2006), también es transversal, puesto que la toma de información se limitó a una sola ocasión. Es, además, no experimental, porque se obtiene y analiza la información sin modificar ninguna de las condiciones existentes.

El instrumento que se aplicó para la presente investigación fue utilizado por Yeung et al. (2000). Se trata entonces de un cuestionario probado y validado tanto en el estudio de estos autores como en los de otros que también lo han aplicado (Gómez, 2008). Consta de 58 reactivos. Usa una escala tipo Likert de seis opciones, que ascienden gradualmente de la siguiente manera: "no sé/no aplica, muy poco, poco, algo, mucho y muchísimo".

El objeto del presente estudio es la industria fabricante de celulosa, cartón y papel de México. Para determinar el universo se utilizó el directorio 
de la Cámara del Papel, la institución que agremia a los principales fabricantes de papel, de empaque y de cartón. Esta entidad conjunta a veintisiete socios, dos agremiados y tres cooperadores, y tiene presencia en veinte estados de México. Dentro de sus socios se encuentran firmas de reconocido prestigio como Kimberly-Clark de México, BioPappel, Procter \& Gamble, Copamex y Cartón Ponderosa, entre otros.

Se contactó a los directivos de cada empresa para invitarlos a participar en el proyecto, se les explicó luego el objetivo y alcance del estudio y se entregaron los cuestionarios que deberían contestar sus directores, gerentes, jefes de departamentos y supervisores (mandos medios, gerenciales y directivos). Estos actores fueron el sujeto de estudio. Al final se rescataron 420 cuestionarios, provenientes de veinticinco empresas que lo contestaron. Estas tienen presencia en quince estados de México.

La dificultad para poder recopilar los cuestionarios en mención se hizo presente. Esto pues los sujetos de observación "no están acostumbrados a ser observados y mucho menos a contestar cuestionarios que puedan poner en tela de juicio los procedimientos o la manera de hacer las cosas", según lo explicó el director de una de las plantas, en donde des- afortunadamente solo se rescató el $20 \%$ de los cuestionarios enviados. Como consecuencia de lo anterior se tomó la decisión de trabajar con todos los cuestionarios recuperados.

Para tal efecto los datos se trabajaron en el programa estadístico SPSS versión 19.0. Se calculó la confiabilidad del instrumento por medio de la prueba Alfa de Cronbach, cuyo resultado se pueden apreciar en la tabla 1. De acuerdo con Barraza Macías (2007), un valor del alfa de Cronbach menor a 0,60 es mediocre, de 0,60 a 0,65 es indeseable, de 0,65 a 0,70 es aceptable, de 0,70 a 0,80 es respetable y de 0,80 a 0,90 es muy bueno. Es necesario destacar que esta prueba se corrió para cada dimensión (estilos de aprendizaje organizacional e incapacidades de aprendizaje organizacional).

Tabla 1. Confiabilidad del instrumento de medición

\begin{tabular}{|c|l|c|}
\hline $\begin{array}{c}\text { Prueba } \\
\text { utilizada }\end{array}$ & Dimensión & Valor obtenido \\
\hline \multirow{2}{*}{$\begin{array}{l}\text { Alfa de } \\
\text { Cronbach }\end{array}$} & $\begin{array}{l}\text { Estilos de } \\
\text { aprendizaje }\end{array}$ & 0,868 \\
\cline { 2 - 3 } & $\begin{array}{l}\text { Incapacidades } \\
\text { de aprendizaje }\end{array}$ & 0,784 \\
\hline
\end{tabular}

Fuente: Elaboración propia.

\section{RESULTADOS}

Para obtener el diagnóstico de los estilos de aprendizaje y de las inca- 
pacidades en principio se obtuvieron los promedios y frecuencia para cada indicador, en cada uno de los tipos de los estilos de aprendizaje (ver tabla 2) y de las incapacidades (ver tabla 3). Esto muestra una descripción del tipo de estilo y de las incapacidades las empresas estudiadas.

Tabla 2. Estilos de aprendizaje: promedios y frecuencia

\begin{tabular}{|l|c|c|}
\hline \multicolumn{1}{|c|}{ Estilos de aprendizaje } & Promedio & Frecuencia \\
\hline Mejora continua & 4,1 & 183 \\
\hline Marcas de referencia & 3,4 & 44 \\
\hline Experimentadoras & 3,9 & 150 \\
\hline Adquisición de competencias & 3,4 & 45 \\
\hline
\end{tabular}

Fuente: Elaboración propia.

Tabla 3. Incapacidades de aprendizaje: promedios y frecuencia

\begin{tabular}{|l|c|c|}
\hline Incapacidades de aprendizaje & Promedio & Frecuencia \\
\hline Ceguera & 3,86 & 222 \\
\hline Candidez & 3,53 & 51 \\
\hline Homogeneidad & 2,89 & 13 \\
\hline Acoplamiento estrecho & 2,98 & 20 \\
\hline Parálisis & 3,30 & 47 \\
\hline Aprendizaje de supersticiones & 3,03 & 42 \\
\hline Difusión deficiente & 3,46 & 27 \\
\hline
\end{tabular}

Fuente: Elaboración propia.

Es importante saber si existen diferencias estadísticamente significativas entre los grupos correspondientes a cada tipo de estilos de aprendizaje y de las incapacidades. Para tal efecto se aplicó un análisis de la varianza con una prueba $F$. Esta, de acuerdo con Lind, Marchal y Wathen (2005), se utiliza con el fin de probar si dos muestras provienen de poblaciones que tienen varianzas iguales (la cual se considera hipótesis nula).

Los resultados de este análisis se muestran en las tablas 4 y 5 . En la primera columna se incorporan los tipos de cultura, en la segunda se indican las fuentes de varianza, en la 
tercera, la suma de los cuadrados ya calculados, en la cuarta se disponen los grados de libertad asociados a cada fuente de varianza, en la quinta se presenta el cuadrado de la media, en la sexta se insertan los resultados de la prueba $\mathrm{F}$ y, en la séptima, el nivel de significación que muestra el peso de la evidencia contra la hipótesis nula.

Tabla 4. Análisis de varianza con un factor: estilos de aprendizaje

\begin{tabular}{|l|l|c|c|c|c|c|}
\hline 1 & \multicolumn{1}{|c|}{2} & 3 & 4 & 5 & 6 & 7 \\
\hline \multirow{4}{*}{ Mejora continua } & $\begin{array}{c}\text { Suma de } \\
\text { cuadrados }\end{array}$ & Gl & $\begin{array}{c}\text { Media } \\
\text { cuadrática }\end{array}$ & F & Sig. \\
\hline & Entre grupos & 240862 & 274 & 0,879 & 5892 & 0,0 \\
\cline { 2 - 7 } & Intra grupos & 20142 & 135 & 0,149 & & \\
\hline \multirow{3}{*}{$\begin{array}{l}\text { Marcas de } \\
\text { referencia }\end{array}$} & Total & 261004 & 409 & & & \\
\hline \multirow{3}{*}{\begin{tabular}{l} 
Experimentadoras \\
\cline { 2 - 8 }
\end{tabular}} & Entre grupos & 390283 & 274 & 1,424 & 5180 & 0,0 \\
\cline { 2 - 8 } & Intra grupos & 37125 & 135 & 0,275 & & \\
\cline { 2 - 8 } & Intra grupos & 31131 & 135 & 0,231 & & \\
\hline \multirow{3}{*}{$\begin{array}{l}\text { Adquisición de } \\
\text { competencias }\end{array}$} & Total & 424736 & 409 & & & \\
\cline { 2 - 8 } & Entre grupos & 305973 & 274 & 0,117 & 4090 & 0,0 \\
\cline { 2 - 8 } & Intra grupos & 36857 & 135 & 0,273 & & \\
\hline
\end{tabular}

Fuente: Elaboración propia.

Tabla 5. Análisis de varianza con un factor: incapacidades de aprendizaje

\begin{tabular}{|c|c|c|c|c|c|c|}
\hline 1 & 2 & 3 & 4 & 5 & 6 & 7 \\
\hline & & $\begin{array}{l}\text { Suma de } \\
\text { cuadrados }\end{array}$ & Gl & $\begin{array}{c}\text { Media } \\
\text { cuadrática }\end{array}$ & $\mathrm{F}$ & Sig. \\
\hline \multirow{3}{*}{ Mejora continua } & Entre grupos & 240862 & 274 & 0,879 & 5892 & 0,0 \\
\hline & Intra grupos & 20142 & 135 & 0,149 & & \\
\hline & Total & 261004 & 409 & & & \\
\hline \multirow{3}{*}{$\begin{array}{l}\text { Marcas de } \\
\text { referencia }\end{array}$} & Entre grupos & 390283 & 274 & 1,424 & 5180 & 0,0 \\
\hline & Intra grupos & 37125 & 135 & 0,275 & & \\
\hline & Total & 427408 & 409 & & & \\
\hline
\end{tabular}




\begin{tabular}{|c|l|c|c|c|c|c|}
\hline 1 & \multicolumn{1}{|c|}{2} & 3 & 4 & 5 & 6 & 7 \\
\hline \multirow{4}{*}{ Experimentadoras } & & $\begin{array}{c}\text { Suma de } \\
\text { cuadrados }\end{array}$ & Gl & $\begin{array}{c}\text { Media } \\
\text { cuadrática }\end{array}$ & F & Sig. \\
\cline { 2 - 7 } & Entre grupos & 393605 & 274 & 1,437 & 6229 & 0,0 \\
\cline { 2 - 8 } & Tntra grupos & 31131 & 135 & 0,231 & & \\
\hline \multirow{3}{*}{$\begin{array}{l}\text { Adquisición de } \\
\text { competencias }\end{array}$} & Entre grupos & 305973 & 274 & 1,117 & 4090 & 0,0 \\
\cline { 2 - 8 } & Intra grupos & 36857 & 135 & 0,273 & & \\
\cline { 2 - 8 } & Total & 342831 & 409 & & & \\
\hline
\end{tabular}

Fuente: Elaboración propia.

Tabla 6. Resumen del modelo de regresión por variable

\begin{tabular}{|l|c|c|c|c|c|c|c|c|c|c|c|c|}
\hline $\begin{array}{c}\text { Variable } \\
\text { dependiente }\end{array}$ & \multicolumn{2}{|c|}{ Experimentadoras } & \multicolumn{3}{c|}{$\begin{array}{c}\text { Marcas de } \\
\text { referencia }\end{array}$} & \multicolumn{2}{c|}{$\begin{array}{c}\text { Adquisición de } \\
\text { competencias }\end{array}$} & \multicolumn{3}{c|}{ Mejora continua } \\
\hline $\mathbf{1}$ & $\mathbf{2}$ & $\mathbf{3}$ & $\mathbf{4}$ & $\mathbf{5}$ & $\mathbf{6}$ & $\mathbf{7}$ & $\mathbf{8}$ & $\mathbf{9}$ & $\mathbf{1 0}$ & $\mathbf{1 1}$ & $\mathbf{1 2}$ & $\mathbf{1 3}$ \\
\hline $\begin{array}{l}\text { Variable } \\
\text { independiente }\end{array}$ & $\mathrm{r}^{2}$ & $\mathrm{~B}$ & $\mathrm{Sig}$. & $\mathrm{r}^{2}$ & $\mathrm{~B}$ & Sig. & $\mathrm{r}^{2}$ & $\mathrm{~B}$ & Sig. & $\mathrm{r}^{2}$ & $\mathrm{~B}$ & Sig \\
\hline Ceguera & 0,424 & 0,670 & 0,000 & 0,389 & 0,643 & 0,000 & 0,345 & 0,543 & 0,000 & 0,380 & 0,498 & 0,000 \\
\hline Candidez & 0,122 & 0,483 & 0,000 & 0,135 & 0,509 & 0,000 & 0,122 & 0,434 & 0,000 & 0,151 & 0,422 & 0,000 \\
\hline Homogeneidad & 0,024 & 0,166 & 0,002 & 0,062 & 0,265 & 0,000 & 0,024 & 0,147 & 0,002 & 0,014 & 0,100 & 0,015 \\
\hline $\begin{array}{l}\text { Acoplamiento } \\
\text { estrecho }\end{array}$ & $-0,002$ & $-0,011$ & 0,866 & 0,005 & 0,093 & 0,137 & $-0,002$ & $-0,005$ & 0,926 & 0,003 & $-0,053$ & 0,280 \\
\hline Parálisis & 0,000 & 0,024 & 0,746 & 0,012 & 0,162 & 0,030 & 0,001 & 0,039 & 0,564 & 0,005 & 0,082 & 0,165 \\
\hline $\begin{array}{l}\text { Aprendizaje } \\
\text { supersticiones }\end{array}$ & 0,026 & $-0,169$ & 0,001 & 0,002 & $-0,049$ & 0,344 & 0,019 & $-0,138$ & 0,003 & 0,024 & $-0,129$ & 0,002 \\
\hline $\begin{array}{l}\text { Difusión } \\
\text { deficiente }\end{array}$ & 0,148 & 0,542 & 0,000 & 0,139 & 0,527 & 0,000 & 0,177 & 0,532 & 0,000 & 0,172 & 0,458 & 0,000 \\
\hline
\end{tabular}

Fuente: Elaboración propia.

Como se observa, la diferencia de medias es significativa en todos los grupos. Esto implica que están diferenciados de acuerdo con el procedimiento estadístico aplicado, por lo que son confiables los resultados mostrados en las tablas 3 y 4 . Posteriormente, con el propósito de contrastar las hipótesis de estudio se aplicaron modelos de regresión lineal usando el procedimiento de mínimos cuadrados ordinarios. Esto para eva- 
luar la relación lineal de dependencia que, de acuerdo con Hair, Anderson, Tatham y Black (2006), es la técnica de dependencia más versátil y utilizada, aplicable en cualquier ámbito de los negocios. Según ellos, esta puede ser empleada para analizar la relación entre una variable criterio y una o varias variables independientes.

Cabe destacar que los modelos de regresión se aplicaron de la siguiente manera: se contrastaron cada uno de los cuatro tipos de estilos de aprendizaje con cada una de las siete incapacidades. Las variables independientes fueron las incapacidades. Esto generó un conjunto de veinti- ocho modelos (siete tipos de incapacidades por cuatro estilos de aprendizaje). Los resultados se muestran en la tabla 6.

Se asume, de acuerdo con la literatura revisada, como son Choo (1999), Gómez (2008), Guzzeta (2008), Hernández (2004) y Wang (2011), que coinciden en que existe una relación entre las incapacidades de aprendizaje organizacional y los estilos de aprendizajeorganizacional.Conbase en lo anterior, este modelo incluye la variable independiente 'incapacidades de aprendizaje organizacional', como explicativa de los estilos de aprendizaje organizacional, así:

$$
\mathrm{Y}_{\text {estilos de aprendizaje }}=\alpha+\mathrm{b}_{1} \mathrm{X}_{\text {incapacidades de aprendizaje }}+€
$$

En donde:

$\mathrm{Y}_{\text {Ede A }}=$ Estilos de aprendizaje organizacional

$$
\alpha=\text { Intercepto }
$$

$\mathrm{X}_{\text {Inc. de ap. }}=$ Incapacidades de aprendizaje organizacional

$$
€=\text { Término de error }
$$

$\mathrm{b}_{1}$, = Coeficiente asociado a la variable independiente

Con los siguientes resultados:

$$
\begin{gathered}
\mathrm{Y}_{\mathrm{EdeA}}=1.999+.541 \mathrm{X}_{\text {Inc. de ap }} \\
(8.816)(8.245) \\
\mathrm{R}^{2}=.143
\end{gathered}
$$


Resulta de este modo estadísticamente significativo el impacto de la variable independiente 'incapacidades de aprendizaje organizacional' sobre los estilos de aprendizaje organizacional. Es importante resaltar entonces que los objetivos del análisis se cumplen cabalmente al evaluar la significancia estadística de la variable independiente sobre la variable dependiente con el estadístico $\mathrm{t}$ de student y su p-valor asociado de 0,000 .

\section{DISCUSIÓN Y CONCLUSIONES}

Este trabajo se realizó con el fin de indagar la posible relación entre las incapacidades de aprendizaje y los estilos de aprendizaje organizacional en la industria papelera, cartonera y celulosa de la República Mexicana, así como de responder a la pregunta de investigación y testear las hipótesis de trabajo correspondientes.

Con base en los resultados estadísticos obtenidos, es posible afirmar que estadísticamente hablando existe evidencia de una relación significativa entre las incapacidades de aprendizaje y los estilos de aprendizaje organizacional. Esto confirma lo indicado al interior de la literatura analizada.

Argyris (1999) y Gómez, Villarreal y Villarreal (2015) coinciden al afirmar que todas las organizaciones generan en mayor o menor medida conocimiento organizacional, estén conscientes de esto o no y estén preparadas para ello o no. Afirman que las incapacidades impiden que el conocimiento se genere y se generalice de mejor manera. De acuerdo con los resultados obtenidos, es posible afirmar que las incapacidades de aprendizaje influyen en los estilos de aprendizaje organizacional que las organizaciones estudiadas usan para resolver sus problemas y para dar respuesta a las demandas de sus clientes.

Según Gómez (2008), no se debe olvidar que la presencia de una sola incapacidad puede ser suficiente para disminuir e incluso bloquear el aprendizaje en una organización. Las incapacidades son, de acuerdo con este autor, como enfermedades silenciosas que invaden a la organización sin que sus dueños o gerentes se den cuenta de lo que sucede. Cuando estos logran percatarse de esta puede ser ya demasiado tarde, pues la organización podría estar altamente infectada para ese momento, lo que hace que el estilo de aprendizaje se afecte también.

A pesar de que el sector estudiado, por su naturaleza, es altamente competitivo y, como consecuencia, los directores y gerentes de las diferentes 
plantas son académica y técnicamente competentes, temas como las incapacidades de aprendizaje y cómo estas pueden afectar los estilos de aprendizaje de las empresas, por lo general, han sido ajenos a su bagaje cultural y son desconocidos para ellos. Sin embargo, dichos actores, en la práctica, ven sus efectos y consecuencias, pues estos aspectos terminan por volver ineficientes a sus empresas e incluso, en ocasiones, ocasionando su cierre. A pesar de lo anterior, estos directivos no logran vislumbrar, por lo general, qué es lo que provoca las ineficiencias en los procesos productivos o administrativos de sus fábricas y solo sufren las consecuencias.

Vale la pena entonces hacer en este punto una reflexión, pues de la forma en la que aprenden las empresas depende en primer término cómo estas descubren problemas y cómo desarrollan soluciones para mantenerse vigentes en el negocio y prosperar en él. Es necesario que los líderes de las empresas estén enterados de estos temas, pues son vitales para el desarrollo de cualquier negocio, en particular si este se desenvuelve en un ambiente altamente competitivo, como es el caso del que ha sido considerado en el presente estudio.

En relación con el primer objetivo específico de la investigación —así como con su correspondiente pregunta de investigación e hipótesis-, es decir, determinar qué estilo de aprendizaje organizacional se presenta con más intensidad en las empresas estudiadas, se encuentra que, con base en los cálculos estadísticos realizados, es posible concluir que la mejora continua es el estilo de aprendizaje dominante en la industria papelera, cartonera y de la celulosa en México. Esto habla de una manera particular de realizar el trabajo cotidiano por parte de la industria estudiada, que consiste en dominar una forma de hacer las cosas hasta que se tiene control sobre todas las variables de la producción y la comercialización. Entonces se inicia un proceso de mejoramiento de cada una de las actividades que se llevan a cabo, realizado estos cambios paso a paso.

Algo paradójico se presenta aquí, pues el estilo de aprendizaje dominante en la industria estudiada es propio de empresas que enfrentan mercados estables, sin cambios significativos y con una competencia moderada, pero las características del mercado en el que se encuentran las compañías estudiadas son más bien la volatilidad y la alta competitividad. Tal vez la explicación de lo anterior se encuentre en que este sector de la industria generalmente está sujeto a un sinnúmero de reglamen- 
taciones tanto fiscales, financieras y contables como ambientales. Estas hacen que las decisiones que se toman en los diferentes temas sean tratadas con tacto y, en cada caso, procurando medir el riesgo que cualquier cambio puede significar.

Para dar respuesta al segundo objetivo específico de la investigación - así como con su correspondiente pregunta de investigación e hipótesis-, a saber: determinar qué incapacidad se presenta con más intensidad en las empresas estudiadas, se encuentra que, con base en los cálculos estadísticos obtenidos, es posible afirmar que la 'ceguera' es la incapacidad que con mayor frecuencia se detecta, generalmente cuando la empresa se preocupa, en lo fundamental, por sí misma, de manera endógena. En este caso se termina teniendo ceguera de taller, pues los problemas absorben tanto la energía como la atención y se termina olvidando, entre otros aspectos importantes, la volatilidad del entorno. Este es un factor determinante para que los negocios lleguen a cerrar o no obtengan los resultados esperados.

Lo anterior parecería que no es propio del tipo de empresas que fueron estudiadas, pero hay que tener en cuenta que la ceguera, acompañada de la candidez (la segunda incapacidad en intensidad), lleva a los responsables de las empresas a no evaluar en todos sus alcances la competencia ni el entorno en donde esta se desenvuelve. Ello pone en un gran riesgo la presencia de la compañía en el mercado.

Es necesario resaltar que la presencia de las incapacidades de aprendizaje en las organizaciones es algo que debe preocupar a los dueños, gerentes y estudiosos del aprendizaje organizacional. Estas impiden que el conocimiento se genere de mejor manera en las empresas. Desafortunadamente, como a primera vista este tema pareciera no estar ligado de manera directa a aspectos tan visibles como la productividad o la rentabilidad de las empresas, por lo general pasa a ser inadvertido ante los responsables de las empresas. Estos terminan sufriendo, sin saber exactamente por qué, las consecuencias de las deficiencias existentes en materia de aprendizaje organizacional.

Como resultado del análisis de la tabla 3 , en particular, en donde se observa que la ceguera es la incapacidad que se presenta con mayor intensidad en todos los estilos de aprendizaje del sector estudiado, es posible llegar a una de las conclusiones de la presente investigación, a saber: la deficiencia de no evaluar de manera correcta las amenazas y 
oportunidades del entorno es el problema más importante del que adolece la industria estudiada y que, independientemente de que se esté en un sector competitivo, por esta causa, se está dejando de lado un sinfín de oportunidades que no se están aprovechando. Planteado de otra manera, solo con el hecho de que algunos de los integrantes del sector estudiado pudieran ser conscientes de las deficiencias de aprendizaje organizacional que padecen y trabajaran para tratar de superarlas podría ser posible que se instalaran en una posición de ventaja competitiva más conveniente que la que tienen sus principales competidores.

Como consecuencia de lo anterior, resulta necesario tratar este tema y hacer consientes de la importancia del mismo a los directivos y gerentes de estas empresas. Ello con el fin de que conozcan las causas de las deficiencias organizacionales que los aquejan y, con ello, no solo les sea posible superarlas, sino que también, como sector, puedan llegar a ser más competitivos. Esto, en particular, en la actualidad, cuando México, como otros países de la región, requiere no solo ser más eficaz y productivo, sino también más competitivo.

Las dificultades más destacadas a las que se enfrentó el equipo investigador para realizar este trabajo estuvieron principalmente en la existencia de una limitada colaboración por parte los encuestados y en su renuencia a proporcionar datos que, de acuerdo con la percepción de los sujetos de observación, no eran relevantes pues, en apariencia, no se reflejan ni en los índices de productividad ni en la rentabilidad.

Derivando de la presente investigación quedan algunas interrogantes pendientes para ser abordadas en estudios posteriores. Se destaca, en particular, la necesidad de analizar la relación entre la cultura organizacional, los estilos de aprendizaje y las incapacidades de aprendizaje. Esto puede contribuir de manera más completa y profunda a la explicación de las variables aquí estudiadas o, incluso, a vincular estas con otras, tales como la supervivencia, el tamaño de la empresa o, entre otros asuntos relevantes, las estrategias de comercialización.

Tal vez el aporte teórico más relevante de este trabajo es la verificación de la influencia de las incapacidades en los estilos de aprendizaje, lo que refuerza, entre otros, el estudio realizado por Yeung et al. (2000). Además, esta investigación constituye un insumo adicional para los procesos de toma de decisiones, vinculados con esta materia, en las organizaciones. 


\section{REFERENCIAS}

Aldrich, R., Llauro, X., Puig, J., Mutje, P., \& Pélach, M. À. (2013). Allocation of carbon dioxide emissions from key production steps in high-grade paper mills. TAPPI JOURNAL, 12(8), 19-28.

Argyris, C. (1993). Cómo vencer las barreras organizativas. Madrid: Díaz de Santos.

Argyris, C. (1999). Sobre el aprendizaje organizacional. México: Oxford University Press.

Barraza Macías, A. (2007). ¿Cómo valorar un coeficiente de confiabilidad? Investigación educativa duranguense, 6, 6-10.

Bravo, E. (2012). Globalización, innovación tecnológica y pobreza. Aproximación a las nuevas conceptualizaciones en Latinoamérica. Espacio abierto: Cuaderno venezolano de sociología. 21(3) 128-145

Beazley, H., Boenisch, J., \& Harden, D. (2003). Knowledge continuity: The new management function. Journal of Organizational Excellence, 22(3), 65-81.

Cámara del Papel (2013). Trabajos de la Sección Técnica. Recuperado de http://www.camaradelpapel.mx/trabajos-de-la-seccion-tecnica/

Carrión Carranza, C. (2007). Educación para una sociedad del conocimiento. México: Trillas.
Choo Wei, C. (1999). La organización inteligente: el empleo de la información para dar significado, crear conocimiento y tomar decisiones. México: Oxford University Press.

De la Madrid, E. (2011). La situación de la industria de la celulosa y el papel en el mundo (publicaciones de Financiera Rural). Recuperado de http://www.financierarural. gob.mx/informacionsectorrural/ Documents/Articulos\%20FR/ Microsoft $\% 20$ Word $\% 20 \% 20$ art $\%$ C3\%ADculo\%20Celulosa\%20y\%20Papel.pdf

Etkin, J. (2007). La capacidad de gobernar en organizaciones complejas. Los acuerdos, la tensión creativa y tolerancia a la diversidad. Cali: Editorial Universidad del Valle.

Geranmayeh, A. (1992). Organizational learning through interactive planning: Design of learning systems for ideal-seeking organizations (Tesis doctoral inédita, Universidad de Pensilvania, Pensilvania, Estados Unidos).

Gómez Romero, J. G. I. (2008). La generación de conocimiento organizacional en la micro, pequeña ymediana empresa (Mipymes) de Durango. Durango: UJED.

Gómez Romero, J. G. I., Villarreal Solis, F. M., \& Villarreal Solís, M. D. (2015, en imprenta). Las incapacidades de aprendizaje 
organizacional y su relación con los estilos de aprendizaje organizacional en la mipyme de la ciudad de Durango. Contaduría y administración. Recuperado de http://www.contaduriayadministracionunam.com.mx/userFiles/ app/pp_2811201353.pdf

Hair, J. F., Tatham, R. L., Anderson, R. E., \& Black, W. (2006). Multivariate data analysis (Vol. 6). Upper Saddle River: Pearson Prentice Hall.

Hernández Silva, F., \& Martí Lahera, Y. (2006). Conocimiento organizacional: la gestión de los recursos y el capital humano. México: ACIMED.

Hernández, R, Fernández, C., \& Baptista, P., (2006), Metodología de la investigación. México: McGraw-Hill.

Huntington, E. (2001). La tercera vía. México: Diana.

Lenz, H. (1990) Historia del papel en México y cosas relacionadas, 1525-1950. México: Porrúa.

Levitt, B., \& March, J. G. (1988). Organizational learning. Annual Review of Sociology, 14, 319-340. Lind, D. A., Marchal, W. G., \& Wathen, S. A. (2005). Statistical techniques in business and economics. Irwin: McGraw-Hill.

Lounamaa, P., \& March, J. G. (1987). Adaptative coordination of a learning team. Management Science, 33(1), 107-123.
Nonaka, I., \& Takeuchi, H. (2000). La organización creadora de conocimiento: cómo las compañías japonesas crean la dinámica de la $i$. México: Oxford University Press.

PriceWaterhouseCoopers (2011). Global forest, paper \& packaging industry survey, 2011 edition - survey of 2010 results. Recuperado de www.pwc.com/fpp

Prieto Pastor, I. M. (2004). Una valoración de la gestión del conocimiento para el desarrollo de la capacidad de aprendizaje en las organizaciones: propuesta de un modelo integrador (Tesis de doctorado inédita) Universidad de Valladolid, Valladolid, España.

Probst, G. J. B., \& Büchel, B. S. T. (1995). La pratique de l'entreprise apprenante. París: Les Éd. d'Organisation.

Probst, G., Raub, S., \& Romhardt, K. (2001). Administre el conocimiento: los pilares del éxito. México: Prentice Hall.

Puerto Becerra, P. (2010). La globalización y el crecimiento empresarial a través de estrategias de internacionalización. Pensamiento \& Gestión, 28, 171-195.

Redacción Teorema Ambiental (2013). La industria del papely de la celulosa. Recuperado de http:// www.teorema.com.mx/biodiversidad/forestal/mexico-aprovecha-el-uso-forestal-sustentable/ 
Senge, P. (1993). La quinta disciplina. México: Granica.

Toffler, A. (1999). El cambio del poder. Barcelona: Plaza \& Janes.

Valdés, L. (2002). La re-evolución empresarial del siglo XXI. Conocimiento y capital intelectual: las nuevas ventajas competitivas de la empresa. Bogotá: Norma.

Vázquez, H., Martínez, F., \& Monroy Alvarado, G. (2007). Más allá del conocimiento: un enfo- que sistémico. Administración y Organizaciones, 10(19), 23-38.

Watkins, K. E., \& Marsick, V. J. (1993). Sculpting the learning organization. Nueva York: Jossey Bass.

Yeung, A., Ulrich, D., Nason, S., \& Von Glinow, M. A. (2000). Las capacidades del aprendizaje en la organización: Cómo aprender a generar ideas con impacto. México: Oxford University Press. 